\title{
Clostridium difficile: An Overview
}

\author{
Jane Murphy \\ Connolly Hospital, Blanchardstown, Dublin, Ireland
}

doi: 10.3396/ijic.V5i2.015.09

\section{Background}

Clostridium difficle is a spore forming anaerobic bacterium that is widely distributed in soil and the intestinal tracts of animals. C. difficile is commonly found in the human intestine where it is carried asymptomatically. The bacterium produces toxins which damage the gut wall. It is mainly a Healthcare associated infection but there is an increasing recognition of community associated cases. The prevalence of asymptomatic $C$. difficile colonisation ranges from less than $5 \%$ in the community to over $20 \%$ of patients in hospital or other healthcare facilities. The spectrum of $C$. difficile human disease ranges from asymptomatic colonisation to potential fatal colitis.

C. difficile associated disease (CDAD) usually presents with symptoms such as profuse foul-smelling diarrhoea, abdominal cramps/pain and fever where the normal gut flora is altered during/following antibiotic therapy. It can present up to 10 weeks after antibiotic therapy. Pseudo membranous colitis (PMC) is the most severe manifestation of the disease. PMC is usually pan colitis, however, a right-sided colitis is also described, featuring fever, pain and decreased gut motility often with only mild diarrhoea. Severely ill patients may have little or no diarrhoea due to toxic megacolon where the inflamed large bowel becomes massively distended with gas and a paralytic ileus that may result from loss of colonic muscular tone. This may lead to perforation of the bowel and can result in death.

The main risk factors associated with CDAD include exposuretoantibiotics, advancedageandhospitalisation. The most commonly reported antibiotics implicated in the development of CDAD are the broad spectrum cephalosporins, fluroquinolones and clindamycin. CDAD predominantly occurs in the older patients, but hospitalisation, recent gastrointestinal surgery or procedures and immunosuppressive therapy may also predispose to infection.

\section{C. difficile ribotype $\mathbf{0 2 7}$}

The United States (US) Centres for Disease Control (CDC) have reported an increase in the incidence of CDAD. C. difficile ribotype 027 (Toxinotype 111) which is a hypervirulent strain was identified in Quebec, Canada. It is characterized by PCR testing. C. difficile ribotype 027 outbreaks have occurred in at least 16 US states. In Europe, C. diffficile ribotype 027 has been recognised in at least 16 hospitals in the Netherlands, 13 in Belgium, 9 in France and 75 in the UK, where there was a large recognised outbreak in Stoke Mandeville hospital in 2005.

Ireland reported their first case of $C$. difficile ribotype 027 recently from a patient transferred from a UK 
hospital. Two clusters of $C$. difficile ribotype 027 have also been reported from two Irish hospitals.

\section{Surveillance}

Surveillance of $C$. difficile varies throughout the world with some countries having mandatory systems in place, such as UK and Ireland, some states in Canada and hospitalised severe cases in France while other countries have a voluntary system such as the U.S., Western Australia, Netherlands and Belgium. The European Centre for Disease prevention and Control (ECDC) have recommended that member states should develop early warning mechanisms and should implement surveillance systems

In England six regional laboratories now provide access to $C$. difficile ribotyping. This service aims to provide timely information which should optimise the management of $C$. difficile at a local level. This $C$. difficile Ribotyping Network for England (CDRNE) will also collect valuable information on antibiotic risk and outcome data.

\section{Diagnosis of $C$. difficile-associated Disease}

The initial diagnosis of CDAD by clinical manifestations is usually made following the onset of diarrhoea which is foul-smelling and often explosive in nature. The diagnosis of CDAD is confirmed with the laboratory detection of $C$. difficile toxins in the diarrhoeal stool specimens.

\section{Treatment}

The initial treatment of symptomatic patients should if possible be to stop precipitating antibiotic therapy. If this is not feasible for clinical reasons antibiotics with a lower propensity to induce CDAD should be substituted. Oral metronidazole is recommended for the initial treatment of non severe CDAD, vancomycin may be considered to be given in consultation with the microbiologist. Patients who develop severe symptoms or patients with recurrent CDAD should be discussed with the microbiologist for prudent antibiotic therapy.

\section{Infection Prevention and Control precautions for the management of CDAD}

The main route of transmission of CDAD is via the hands of the healthcare worker (HCW) but spores from infected patients can survive in the environment for several months. Equipment near the patient, such as commodes, bedpans and patient call bells, can be heavily contaminated. Stringent infection prevention and control measures need to be adhered to by all HCWs.

\section{Patient placement}

Prompt isolation of all patients with confirmed or suspected CDAD in a single room with a clinical sink for hand washing. Ensuite facilities are preferable but if not available a designated toilet or commode should be made available. Standard and transmission based (contact) precautions should be implemented. Isolation with contact precautions may be discontinued when the patient has had at least 48 hours without diarrhoea and has had a formed and normal stool. Standard precautions must be continued.

In an outbreak setting it may be necessary to cohort patients if sufficient single rooms are not available. Cohorted patients should be managed by designated staff to minimise the risk of cross-infection to other patients. The isolation area should have only stock required on a daily basis to avoid having to discard contaminated items on terminal cleaning.

\section{Patient movement and transfer}

The movement and transport of patients with CDAD should be limited to essential purposes only and prior to any movement the receiving department should be made aware of the need to implement contact precautions taking patient confidentiality into account. Transport equipment (wheelchair, stretcher) used for the transfer should be cleaned and decontaminated before use with another patient.

\section{Hand decontamination and protective clothing}

Hand washing with soap (non-antimicrobial or antimicrobial) and water must be performed before and after all patient and equipment contact. The physical action of rubbing and rinsing is the only method of removal of the spores from hands. Alcohol-based hand rubs do not have reliable sporicidal activity and are not recommended as the only hand hygiene measure when caring for confirmed or suspected CDAD patients. PPE (gloves and aprons) should be worn for all interactions and contact with the patient and their environment. 


\section{Environment and equipment decontamination}

The environment of a patient with CDAD can be heavily contaminated with spores which can easily be transmitted onto the hands of the HCW. The environment of all patients with CDAD and all patient care equipment should be thoroughly cleaned with a neutral detergent and decontaminated daily with a sporicidal disinfectant (e.g. hypochlorite solution - 1000ppm available chlorine), paying particular attention to frequently touched sites such as bedrails, bed tables, call bells, TV remote controls and toilets.

Particular attention should be given to immediate cleaning and decontamination of items likely to be faecally contaminated e.g. the under surfaces and hand contact surfaces of commodes and toilets which should be cleaned and decontaminated after each use. All patient equipment should be in good condition to facilitate effective cleaning. Bedpan washers should be in proper working order and should reach a temperature of $80^{\circ}$ for a minimum of 1 minute. Medical devices (e.g. thermometers, stethoscopes) should, where possible, be dedicated to a single patient and disposable items used where available. No additional measures are required for cutlery and crockery.

In the event of an outbreak, the frequency with which environmental cleaning and decontamination is performed should be increased on the affected ward. Following discharge of the patient thorough cleaning and decontamination of the isolation room /cohort ward should be performed. Curtains should be changed and paper towels and toilet paper discarded.

\section{Management of Linen}

All laundry should be treated as infected linen and washed at either $65^{\circ}$ for 10 minutes or $71^{\circ}$ for 3 minutes.
It may be necessary to add 150ppm of chlorine to the final rinse if these temperatures cannot be achieved. Manual sorting or sluicing of soiled linen is not recommended.

\section{Management of Healthcare Waste}

Waste matter soiled with faeces (e.g. incontinence wear, wipes) which is generated from a patient with CDAD should be disposed of as healthcare risk waste following local policy.

\section{Conclusion}

The importance of early isolation of a patient with known or suspected CDAD is the key to proper control and prevention of the transmission of CDAD. Hand washing and strict adherence to standard and transmission based (contact) precautions is paramount to the management of patients with CDAD. Thorough cleaning and decontamination is required as spores may be disseminated in large numbers and may survive in the environment for long periods of time.

\section{Bibliography}

- Surveillance, Diagnosis and Management of Clostridium difficile -associated disease in Ireland. Health Protection Surveillance Centre. Clostridium difficile sub-committee 2008.

- Vonberg RP, Kuijper EJ, Wilcox MH, et al. Infection control measures to limit the spread of Clostridium difficile. Clin Microbiol Infect 2008; 14 Suppl 5: 2-20.

- Wilcox MH, Fawley WN, Wigglesworth N, Parnell P, Verity $\mathrm{P}$, Freeman J. Comparison of the effect of detergent versus hypochlorite cleaning on environmental contamination and incidence of Clostridium difficile infection. I Hosp Infect 2003; 54: 109-114.

- Bouza E, Munoz P, Alonso R. Clinical manifestations, treatment and control of infections caused by Clostridium difficile. Clin Microbiol Infect 2005; 11 Suppl 4: 57-64.

- $\quad$ Epic2: National Evidence-Based Guidelines for Preventing Healthcare-Associated Infections in NHS Hospitals in England. J Hosp Infect 2007; 65 Suppl 1: S1-64. 